\title{
Calycosin and Genistein Induce Apoptosis by Inactivation of HOTAIR/p-Akt Signaling Pathway in Human Breast Cancer MCF-7 Cells
}

\author{
Jian Chen ${ }^{a}$ Changrong Lin ${ }^{b} \quad$ Wang Yong $^{a} \quad Y_{\text {Y Ye }}^{c} \quad$ Zhaoquan Huang ${ }^{d}$ \\ aSchool of Basic Medical Sciences, Guilin Medical University, Guilin, ${ }^{b}$ Department of General Surgery, \\ Jiangbin Hospital of Guangxi Zhuang Autonomous Region, Nanning, 'Department of Emergency, First \\ Affiliated Hospital of Guangxi Medical University, Nanning, dDepartment of Pathology, Guilin Medical \\ University, Guilin, China
}

\section{Key Words}

Calycosin • Genistein apoptosis $•$ HOTAIR $・$ p-Akt

\begin{abstract}
Background: Calycosin and genistein are the two main components of isoflavones. Previously, we reported that these compounds display antitumor activities in the breast cancer cell lines MCF-7 and T47D. In the present study, we investigated the mechanism of action of calycosin and genistein, and their respective efficacies as potential therapies for the treatment of breast carcinoma in the clinic. Methods: MCF-7 cells were treated with calycosin or genistein. Cell proliferation and apoptosis were measured using CCK8 assay and Hoechst 33258. The expression level of phosphorylated Akt protein was determined by western blotting. Expression level of HOTAIR was quantified by real-time PCR. Results: Both calycosin and genistein inhibited proliferation and induced apoptosis in MCF-7 breast cancer cells, especially after treatment with calycosin. Treatment of MCF-7 cells with calycosin or genistein resulted in decreased phosphorylation of Akt, and decreased expression of its downstream target, HOTAIR. Conclusion: Calycosin is more effective in inhibiting breast cancer growth in comparison with genistein, through its regulation of Akt signaling pathways and HOTAIR expression.
\end{abstract}




\section{Introduction}

Breast cancer is one of the most common cancers threatening women. As the second leading cause of cancer-related death in women, it accounts for approximately $15 \%$ of all female cancer-related deaths in the United States [1]. Recent evidence suggests that increasing vegetable and fruit consumption might reduce the risk of breast cancer [2, 3]. Epidemiologic studies have also reported that the incidence of breast cancer in Asian women is much lower than in Western women, presumably due to higher amounts of phytoestrogens in the diet of Asian women. Thus, the relationship between phytoestrogens and breast cancer has become a major focus of recent research.

Phytoestrogens, sometimes called dietary estrogens, are a diverse group of naturally occurring non-steroidal plant compounds that have been proposed as the natural alternatives to estrogen. Because of their structural similarity to estrogen, phytoestrogens can produce estrogenic and/or anti-estrogenic effects. Phytoestrogens are subdivided into four main classes: isoflavones, stilbened, lignans, and coumestans.

Both calycosin and genistein, belonging to the isoflavone class, are commonly consumed in the diet. It has been shown that calycosin and genistein inhibit many types of cancers including breast cancer [4-7] through the regulation of several cell signaling pathways such as the mitogen-activated protein kinases (MAPK), Akt and JAK/STAT pathways [8-10].

Recent evidence suggests that non-coding RNAs (ncRNAs) are involved in many cellular processes. ncRNAs are divided into two major classes based on transcript size: small ncRNAs and long ncRNAs (lncRNAs). LncRNAs are defined as RNA genes larger than 200 nucleotides that have no protein coding potential $[11,12]$. The HOX transcript antisense RNA (HOTAIR) gene is located within the Homeobox C (HOXC) gene cluster on chromosome 12 and encodes s $2.2 \mathrm{~kb}$ lncRNA molecule. Importantly, HOTAIR is over-expressed in several cancers such as breast, colorectal, liver, pancreas, and laryngeal cancer $[13,14]$. In particular, high expression of HOTAIR in breast cancer is a predictor of metastasis and poor outcome [15]. The expression of HOTAIR has been reported to be regulated by the PI3K/Akt signaling pathway [16]. Despite the growing body of evidence that HOTAIR and isoflavones are involved in breast cancer biology, there are no reports about the relationship between isoflavone and HOTAIR in breast cancer.

Based on the above information, we hypothesized that the HOTAIR-Akt mediated pathway may also be involved in the inhibitory effect induced by calycosin and genistein in MCF-7 cells. In the present study, we investigated the underlying mechanisms involved in the inhibitory effect of calycosin and genistein in MCF-7 cells.

\section{Materials and Methods}

\section{Drug}

Calycosin and genistein (purity > 98\%, demonstrated by high-performance liquid chromatography, extracted by Phytomarker Ltd, Tianjin, China) was dissolved in dimethylsulfoxide (DMSO). The stock of calycosin and genistein $(200 \mathrm{mM})$ were stored at $4^{\circ} \mathrm{C}$ for further use.

\section{Cell Culture}

The human breast cancer cell line (MCF-7) was obtained from the Shanghai Institute of Cell Biology (Chinese Academy of Sciences, Shanghai, China), and the cells were cultured at $37^{\circ} \mathrm{C}$ in a humidified atmosphere of 5\% $\mathrm{CO}_{2}$ in RPMI 1640 media (Gibco-BRL, Gaithersburg, MD) containing 10\% fetal bovine serum (FBS; HyClone, Logan, UT), $100 \mathrm{kU} / \mathrm{L}^{-1}$ penicillin, and $100 \mathrm{mg} / \mathrm{L}^{-1}$ streptomycin. For the cell proliferation assay, cells were starved with low-serum medium (containing 0.5\% CS-FBS) for $24 \mathrm{~h}$.

\section{Cell proliferation assay}

MCF-7 cells were trypsinized and seeded at $3 \times 10^{3}$ cells/well in 96 -well plates. After $24 \mathrm{~h}$, various concentrations of calycosin or genistein were added and incubated for another $48 \mathrm{~h}$. Subsequently, $10 \mu \mathrm{L}$ of the Cell Counting Kit-8 (CCK-8; Sigma, USA) solution (5 g/L, dissolved in phosphate buffered saline; PBS) 
Chen et al.: Calycosin and Genistein Induce Apoptosis

was added. Plates were incubated for an additional $4 \mathrm{~h}$. The optical density for each well was measured using a microculture plate reader (Bio-Tek, USA) at a wavelength of $490 \mathrm{~nm}$.

Cell morphology assessment

MCF-7 cells were treated with calycosin or genistein $(80 \mu \mathrm{M})$ for $48 \mathrm{~h}$, washed with PBS, and then fixed with $4 \%$ paraformaldehyde in PBS for $30 \mathrm{~min}$ at room temperature. After fixation, cells were stained with Hoechst 33258 (5 mg/ml), and observed under the inverted light microscope. Apoptotic cells were identified by fragmented and condensed nuclei.

Quantitative Real-time PCR assay ( $($ PCR)

MCF-7 cells were treated with calycosin or genistein $(80 \mu \mathrm{M})$ for $48 \mathrm{~h}$. RNA was extracted using Trizol (Gibco-BRL). cDNA was preparing using 10 ng of RNA and the RevertAid First Strand cDNA Synthesis Kit (Fermentas, Life Sciences, USA). Next, quantification of HOTAIR was measured by qPCR using SYBR Green qPCR Master Mix (Fermentas, Life Sciences, USA) and specific primers for HOTAIR and GAPDH. GAPDH was used as a housekeeping gene to calculate the relative expression levels of HOTAIR.

\section{Western blot analysis}

After cells were treated with calycosin or genistein $(80 \mu \mathrm{M})$ for $48 \mathrm{~h}$, total cell extracts were prepared in ice-cold lysis buffer. The lysates were centrifuged at $12,000 \mathrm{rpm}$ for $10 \mathrm{~min}$, and protein concentrations in the resulting supernatants were determined by Bio-Rad assay kit using bovine serum albumin as the standard. Equal amounts of protein $(40 \mu \mathrm{g} / \mathrm{lane})$ were separated by SDS-PAGE and transferred onto 0.22 $\mu \mathrm{m}$ polyvinylidene difluoride (PVDF) membranes (Bio-Rad Laboratories, USA). The membranes were blocked in TBS-T (Tris-buffered saline, pH 7.6, 0.05\% Tween 20) containing 5\% non-fat dried milk. The membrane were further incubated sequentially with specific antibodies against p-Akt (1:1000), and Akt (1:1000). After three washes, the blots were incubated with appropriate secondary antibodies coupled to horseradish peroxidase at room temperature for $1 \mathrm{~h}$ and then developed using electrochemiluminescence (ECL) western blot detection reagents (Beyotime, China). Expression levels of the proteins were compared with the control based on the relative intensities of the bands.

Statistical analysis

One-way ANOVA followed by Dunnett's multiple comparison test was used for comparison of different treatments using SPSS 16.0 software. Data were expressed as mean \pm SD, and a probability of $p<0.05$ was considered statistically significant.

\section{Results}

Calycosin and genistein inhibit proliferation of human MCF7 breast cancer cells

To evaluate the anti-proliferative effect of isoflavones, human MCF- 7 breast cancer cells were incubated with calycosin or genistein $(0,5,10,20,30,40,60,80,100 \mu \mathrm{M})$ for $48 \mathrm{~h}$, and cell proliferation was measured using the CCK-8 kit. The results demonstrated that both calycosin and genistein significantly inhibited the proliferation of MCF-7 cells $(p<0.05)$ in a dose-dependent manner (Fig. 1). Additionally, we found that calycosin was more effective than genistein in the inhibition of cell proliferation in MCF-7 cells.

\section{Calycosin and genistein induces apoptosis in human MCF-7 breast cancer cells}

Hoechst 33258 was used to determine whether calycosin or genistein can induce apoptosis in MCF-7 cells. Calycosin- or genistein-treated MCF-7 cells were stained with Hoechst 33258 to provide morphological evidence of apoptosis. As expected, we observed bright blue-stained nuclei with condensed chromatin in MCF-7 cells treated with calycosin or genistein, compared with untreated MCF-7 cells (Fig. 2). This data confirmed that calycosin or genistein could induce apoptosis. In brief, these results corroborated the data from the CCK8 assay. This indicates that induction of apoptosis is likely to be the mechanism of calycosin- and genistein-mediated growth inhibition of MCF-7 cells. 


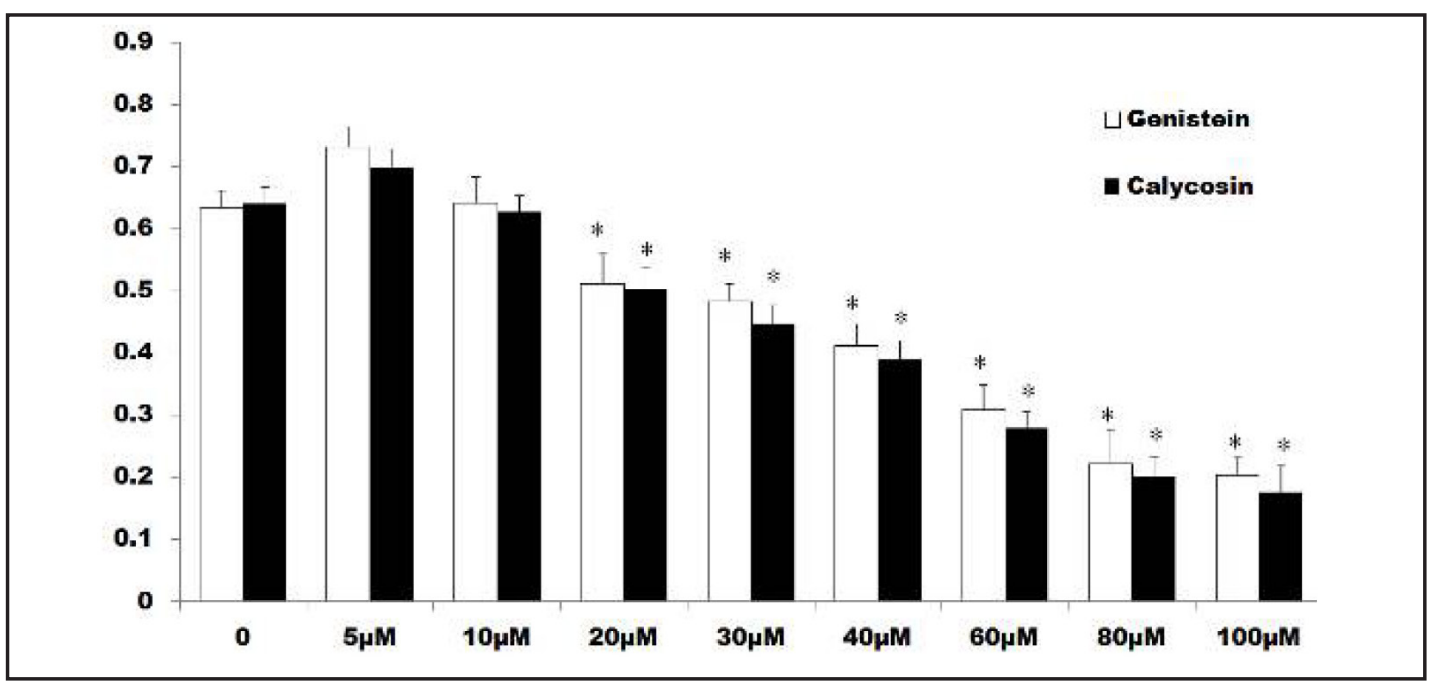

Fig. 1. Antiproliferative effects of calycosin and genistein on human breast cancer cell MCF-7. Cells were treated with various concentrations of calycosin and genistein for $48 \mathrm{~h}$, and then analyzed for viability by CCK8 assay. Data were shown as mean \pm SD. Results are representative of three independent experiments performed in triplicate. Compared with control group $(0 \mu \mathrm{M}){ }^{*} p<0.05$.

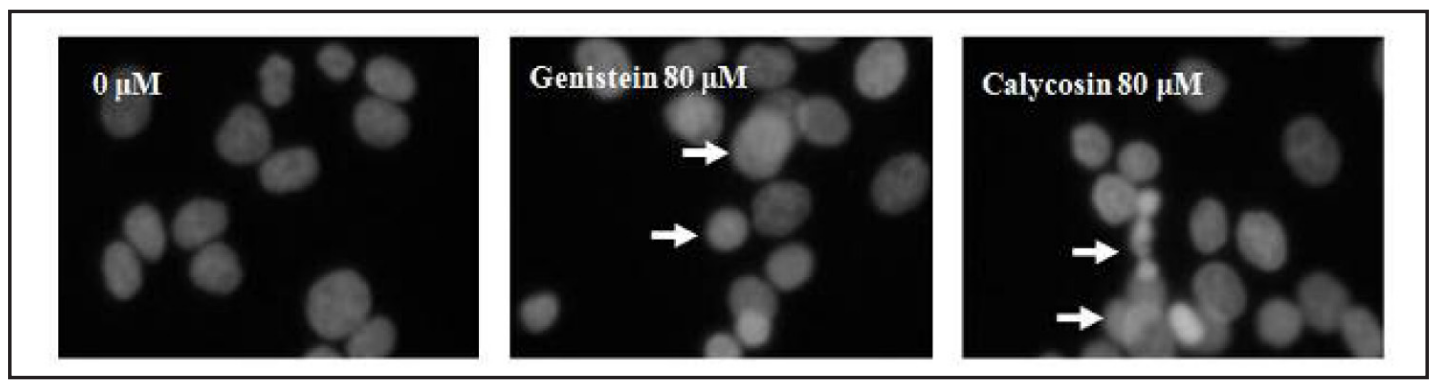

Fig. 2. Calycosin and genistein induced apoptosis in human breast cancer cell MCF-7. Cells were treated with $0 \mu \mathrm{M}, 80 \mu \mathrm{M}$ calycosin or $80 \mu \mathrm{M}$ genistein for $48 \mathrm{~h}$. Morphological changes were observed after staining with Hoechst 33258. The apoptotic cells exhibited fragmented or condensed nuclei (indicated by arrow). These are representative data from three independent experiments.

Calycosin and genistein inactivate Akt in human breast cancer MCF-7 cells

Activation of the phosphatidylinositol 3-kinase (PI3K)/Akt pathway has been widely reported to promote cell survival by inhibiting apoptosis. Therefore, we analyzed the phosphorylation level of Akt in calycosin- or genistein-treated cells by western blot. After treatment of cells with calycosin or genistein, we observed a decrease in the phosphorylation of Akt in MCF-7 cells ( $p$ < 0.05) (Fig. 3). This data suggested that inactivation of the Akt signaling pathway could be responsible for the cell apoptosis induced by calycosin and genistein.

Calycosin and genistein downregulate expression level of HOTAIR in human MCF-7 breast cancer cells

It has been reported that the PI3K/Akt pathway regulates the expression of the oncogenic lncRNA HOTAIR. Since we observed a decrease in activation of the PI3K/Akt pathway, indicated by lower p-Akt levels (Fig. 3), we wanted to see whether HOTAIR levels were effected upon isoflavone treatment. The results showed that calycosin and genistein gradually downregulated expression level of HOTAIR, which was again more apparent in calycosin-treated MCF-7 cells ( $\mathrm{p}<0.05$ ) (Fig. 4). 
Fig. 3. Inhibited Akt proteins expression in in human breast cancer cell MCF-7 by calycosin or genistein. Cells were treated with $0 \mu \mathrm{M}, 80 \mu \mathrm{M}$ calycosin or $80 \mu \mathrm{M}$ genistein for 48 $\mathrm{h}$, and then protein samples were labeled with specific antibodies against Akt and p-Akt. The antibodies to total Akt served as loading controls. There are representative data from three independent experiments. Compared with control group $(0 \mu \mathrm{M}){ }^{*} p<0.05$.

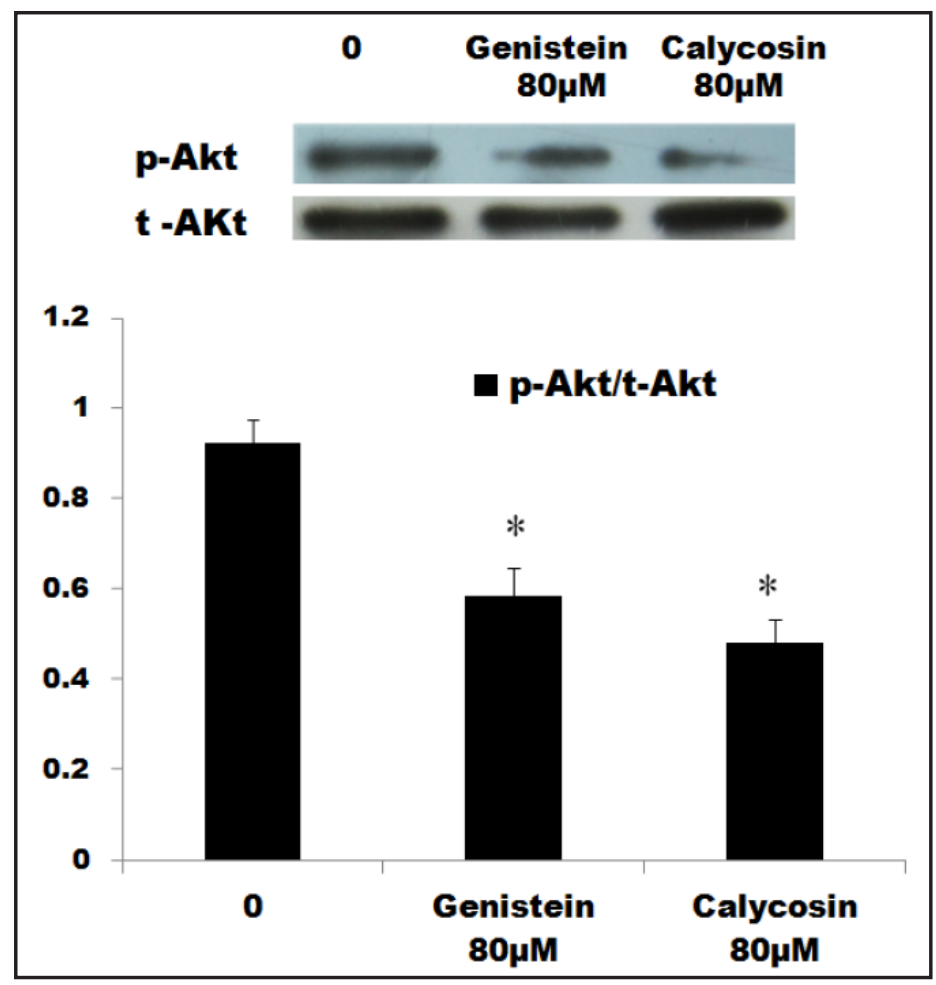

Fig. 4. Expression of HOTAIR after treatment with calycosin $(80 \mu \mathrm{M})$ or genistein $(80 \mu \mathrm{M})$ determined by real-time PCR (mean \pm SD), with GAPDH served as loading controls. Data were obtained from three independent experiments performed in triplicate. Compare with control vehicle $(0 \mu \mathrm{M}) * p<0.05$.

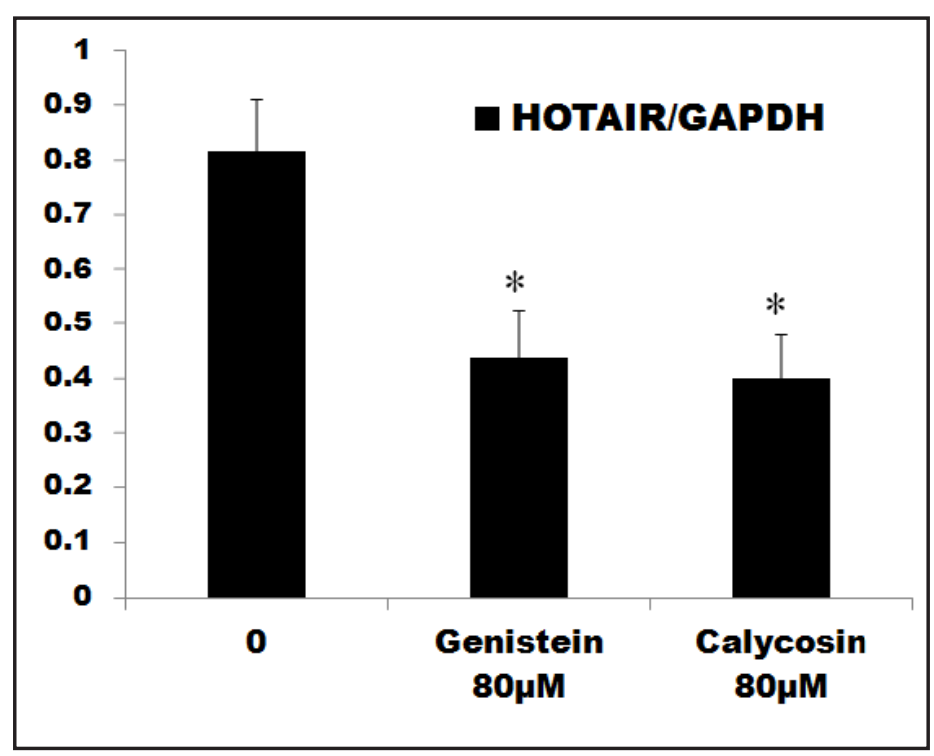

\section{Discussion}

Recently, phytoestrogens have received considerable attention because of their ability to bind estrogen receptors. Isoflavones, lignans, and coumestans comprise the main phytoestrogenic compounds. Isoflavones resemble 17-beta-estradiol in structure, and, as such, are able to bind the estrogen receptor (ER) in vitro, behaving much like a natural selective estrogen receptor modulator (SERM) [17]. Ma et al., reported that isoflavone intake significantly inhibited the development of premenopausal and postmenopausal mammary tumors., indicating the role of phytoestrogens in the prevention and treatment of breast cancer [18].

Calycosin and genistein are two main components of isoflavones that have been attracting attention for their possible anti-cancer effects. Previously, we have reported that 
calycosin significantly inhibited proliferation and induced apoptosis in human breast cancer MCF-7 cells [8]. Furthermore, Prietsch et al., reported that the antitumor effect of genistein involved the modulation of antioxidant enzymes and apoptotic signaling expression, which resulted in apoptosis and autophagy [19]. Based on these findings, we further observed and compared the anti-proliferation effect between calycosin and genistein, so as to provide more valuable information in evaluating the feasibility of their clinical application.

As expected, both calycosin and genistein markedly inhibited cell proliferation of MCF-7 cells in a dose-dependent manner. Moreover, the growth inhibitory effects induced by calycosin were greater than genistein, indicating that calycosin may be more efficacious than calycosin in treating human breast cancer. Furthermore, it was shown that apoptosis is the predominant mode of calycosin- and genistein-mediated cell death, as determined by increased apoptotic cells with Hoechst 33258 measurement.

Akt is a major component of the phosphoinositide 3-kinase (PI3K) signaling pathway. In normal cells, Akt acts as a signal transducer of PI3K and promotes cell proliferation and cell survival. Activation of Akt favors cell survival through the regulation of apoptotic proteins, like the Bcl-2 family and caspases [20,21]. Thus, we believe that the observed downregulation of Akt phosphorylation may explain the mechanism for calycosin- and genistein-induced apoptosis.

The IncRNA HOTAIR is shuttled from chromosome 12 to chromosome 2 by a component of the Polycomb Repressive Complex 2 (PRC2) and represses transcription of homeobox D (HOXD) genes [22]. HOTAIR interacts with both PRC2 and lysine specific demethylase 1 (LSD1) complexes and couples histone H3 lysine 27 methylation and lysine 4 demethylation for epigenetic silencing of not only HOXD genes, but also many other genes. Evidence suggests that HOTAIR regulates key pathways in cancer invasion and metastasis. HOTAIR increased breast cancer invasiveness and metastasis by inducing positive regulators of cancer metastasis (ABL2 SNAIL, LAMB3 and LAMC2) in a manner dependent on PRC2 [14]. Some studies demonstrated that PI3K/AKT signaling influenced the expression of HOTAIR [16]. However, little is known about the relationship between isoflavones, Akt, and HOTAIR in breast cancer cells. This prompted us to investigate whether isoflavones act as a modulator of Akt and HOTAIR, or a related signaling pathway. In the present study, our data revealed that along with reduced p-Akt levels, HOTAIR expression decreased in a dose-dependent manner upon treatment with calycosin or genistein. Therefore, this study is the first to demonstrate that calycosin and genistein regulate HOTAIR in MCF-7 cells.

In conclusion, our results show that calycosin and genistein inhibit breast cancer cell growth through inactivation of the PI3K/Akt signaling pathway, which resulted in decreased expression of the downstream target HOTAIR. These findings improve our understanding of how calycosin and genistein regulate lncRNA in breast cancer and identifies novel targets for breast cancer therapy.

\section{Disclosure Statement}

The authors declare no conflict of interest.

\section{Acknowledgements}

This research was supported by grants from the National Natural Science Foundation of China (NO. 81260343) and the National Natural Science Foundation of Guangxi (2013GXNSFAA019122).

\section{References}

1 Hilakivi-Clarke L, Wang C, Kalil M, Riggins R, Pestell R G: Nutritional modulation of the cell cycle and breast cancer. Endocr Relat Cancer 2004;11:603 -622. 
Chen et al.: Calycosin and Genistein Induce Apoptosis

-2 Jin S, Zhang Q Y, Kang X M, Wang J X, Zhao W H: Daidzein induces MCF-7 breast cancer cell apoptosis via the mitochondrial pathway. Ann Oncol 2010;21:263-268.

-3 Gandini S, Merzenich H , Robertson C, Boyle P: Meta-analysis of studies on breast cancer risk and diet: the role of fruit and vegetable consumption and the intake of associated micronutrients. Eur J Cancer 2000;36:636-646.

-4 Chen J, Liu L, Hou R, Shao Z, Wu Y, Chen X, Zhou L: Calycosin promotes proliferation of estrogen receptor-positive cells via estrogen receptors and ERK1/2 activation in vitro and in vivo. Cancer Letters 2011;308:144-151.

-5 Tian J, Duan YX, Bei CY, Chen J: Calycosin induces apoptosis by upregulation of RASD1 in human breast cancer cells MCF-7. Horm Metab Res 2013;45:593-598.

-6 Hu XJ, Xie MY, Kluxen FM, Diel P: Genistein modulates the anti-tumor activity of cisplatin in MCF-7 breast and HT-29 colon cancer cells. Arch Toxicol 2014;88: 625-635.

7 Pons DG, Nadal-Serrano M, Blanquer-Rossello MM, Sastre-Serra J, Oliver J, Roca P: Genistein modulates proliferation and mitochondrial functionality in breast cancer cells depending on ERalpha/ERbeta ratio. J Cell Biochem 2014;115:949-958.

8 Chen J, Hou R, Zhang X, Ye Y, Wang Y, Tian J: Calycosin Suppresses Breast Cancer Cell Growth via ERbDependent Regulation of IGF-1R, p38 MAPK and PI3K/Akt Pathways. PLoS One 2014;9:e91245,

-9 Chen J, Zhao X, Ye Y, Wang Y, Tian J: Estrogen receptor beta-mediated proliferative inhibition and apoptosis in human breast cancer by calycosin and formononetin. Cell Physiol Biochem 2013;32:1790-1797.

10 Privat M, Aubel C, Arnould S, Communal Y, Ferrara M, Bignon YJ: AKT and p21 WAF1/CIP1 as potential genistein targets in BRCA1-mutant human breast cancer cell lines. Anticancer Res 2010;30:2049-2054.

11 Chen K, Rajewsky N: The evolution of gene regulation by transcription

12 factors and microRNAs. Nat Rev Genet2007;8:93-103.

13 Chiyomaru T, Yamamura S, Fukuhara S, Yoshino H, Kinoshita T, Majid S, Saini S, Chang I, Tanaka Y, Enokida H, Seki N, Nakagawa M, Dahiya R: Genistein inhibits prostate cancer cell growth by targeting miR-34a and oncogenic HOTAIR. PLoS One 2013;8:e70372.

-14 Tsai MC, Manor O, Wan Y, Mosammaparast N, Wang JK, Lan F, Shi Y, Segal E, Chang HY: Long noncoding RNA as modular scaffold of histone modification complexes. Science 2010;329:689-693.

15 Gupta RA, Shah N, Wang KC, Kim J, Horlings HM, Wong DJ, Tsai MC, Hung T, Argani P, Rinn JL, Wang Y, Brzoska P, Kong B, Li R, West RB, van de Vijver MJ, Sukumar S, Chang HY: Long noncoding RNA HOTAIR reprograms chromatin state to promote cancer metastasis. Nature 2010;464:1071-1076

16 Kogo R, Shimamura T, Mimori K, Kawahara K, Imoto S, Sudo T, Tanaka F, Shibata K, Suzuki A, Komune S, Miyano S, Mori M: Long noncoding RNA HOTAIR regulates polycomb-dependent chromatin modification and is associated with poor prognosis in colorectal cancers. Cancer Res 2011;71:6320-6326.

17 Yang G, Zhang S, Gao F, Liu Z, Lu M, Peng S, Zhang T, Zhang F: Osteopontin enhances the expression of HOTAIR in cancer cells via IRF1. Biochim Biophys Acta 2014;1839:837-848.

18 Fritz H, Seely D, Flower G, Skidmore B, Fernandes R, Vadeboncoeur S, Kennedy D, Cooley K, Wong R, Sagar $\mathrm{S}$, Sabri E, Fergusson D: Soy, red clover, and isoflavones and breast cancer: a systematic review. PLoS One 2013;8:e81968.

-19 Ma D, Zhang Y, Yang T, Xue Y, Wang P: Isoflavone intake inhibits the development of 7,12-dimethylbenz(a) anthracene(DMBA)-induced mammary tumors in normal and ovariectomized rats. J Clin Biochem Nutr 2014;54:31-38.

20 Prietsch RF, Monte LG, da Silva FA, Beira FT, Del Pino FA, Campos VF, Collares T, Pinto LS, Spanevello RM, Gamaro GD, Braganhol E: Genistein induces apoptosis and autophagy in human breast MCF-7 cells by modulating the expression of proapoptotic factors and oxidative stress enzymes. Mol Cell Biochem 2014;390:235-242.

21 Paplomata E, O'Regan R: The PI3K/AKT/mTOR pathway in breast cancer: targets, trials and biomarkers. Ther Adv Med Oncol 2014;6:154-166.

22 Ciruelos Gil EM: Targeting the PI3K/AKT/mTOR pathway in estrogen receptor-positive breast cancer. Cancer Treat Rev 2014;40:862-871.

-23 Rinn JL, Kertesz M, Wang JK, Squazzo SL, Xu X, Brugmann SA, Goodnough LH, Helms JA, Farnham PJ, Segal E, Chang HY: Functional demarcation of active and silent chromatin domains in human HOX loci by noncoding RNAs. Cell 2007;129:1311-1323. 